\title{
Letter to the Editor:Timing of diuretics in diuresis renography
}

\author{
Arosh S. Perera Molligoda Arachchige ${ }^{1}$ (D) \\ Received: 28 August 2021 / Accepted: 26 September 2021 / Published online: 1 October 2021 \\ (c) Italian Association of Nuclear Medicine and Molecular Imaging 2021
}

\section{To the Editor:}

We have read with great interest the article by Bäck et al. where they have conducted a systematic literature review in an attempt to understand what the most appropriate point in time is, to administer the diuretic in relation to the radiopharmaceutical in performing a diuresis renography in an adult population to distinguish between an obstruction and prolonged renal drainage [1]. Whilst we do perfectly understand that a meta-analysis could not be conducted by the authors due to the lack of homogeneity in the numerical data, we have certain suggestions and questions for the authors.

First of all, we have observed that the authors have included both children and adult participants in their study. However, the PICO (Population, Intervention, Comparison, Outcome) has defined the population as "Adult patients with the clinical question of obstruction or split renal function". Next, the authors have described different radiopharmaceuticals including ${ }^{99 \mathrm{~m}} \mathrm{Tc}-\mathrm{MAG} 3$ which had been administered in six studies, ${ }^{99 \mathrm{~m}} \mathrm{Tc}-\mathrm{DTPA}$ in five studies, ${ }^{123} \mathrm{I}-\mathrm{OIH}$ in four studies, and ${ }^{99 \mathrm{~m}} \mathrm{Tc}-\mathrm{EC}$ in one study. In addition, in one of the studies, the type of radiopharmaceutical used was unknown [1]. We wonder whether the different half-lives between the radiopharmaceuticals could have been one of the possible reasons for the discrepancies observed between results or conclusions drawn from the different studies by different authors that have been described in the text. If it does affect the outcomes, we believe that it would have been better to classify the results according to the type of radiopharmaceutical used.

Furthermore, the authors have stated that the $\mathrm{F}+0$ method is a more practical and shorter technique than $\mathrm{F}-15$ and therefore considered a better alternative, especially if

Arosh S. Perera Molligoda Arachchige

aroshshavinda.pereramolligodaarachchige@ st.hunimed.eu

1 Department of Biomedical Sciences, Humanitas University, Milan, Italy there is only one opportunity to confirm or exclude obstruction [2]. It is unclear to us what "one opportunity to confirm or exclude" means in this statement.

Finally, the authors have discussed a study by Tartiglione et al. where they have acknowledged that the supine position led to more problems related to filling of the bladder compared to the sitting position [3]. Also here, it is unclear whether the position parameter (supine/sitting) could have been a cause of discrepancy between studies. This is an important piece of information that seems to have been missed when the results were discussed. It would be very much appreciated if the authors could clarify these doubts. Thank you.

Funding This work received no specific grant from any funding agency in the public, commercial, or not-for-profit sectors.

\section{Declarations}

Conflict of interest The authors have no conflicts of interest to declare.

\section{References}

1. Bäck AK, Savvopoulos C, Geijer H (2021) Timing of diuretics in diuresis renography. Clin Transl Imaging

2. Turkolmez S, Atasever T, Turkolmez K, Gogus O (2004) Comparison of three different diuretic renal scintigraphy protocols in patients with dilated upper urinary tracts. Clin Nucl Med 29:154-160

3. Tartaglione G, D'Addessi A, De Waure C, Pagan M, Raccioppi M, Sacco E, Cadeddu C, Vittori M, Bassi PF, Ferretti A, Al-Nahhas A, Rubello D (2013) (99m)Tc-MAG3 diuretic renography in diagnosis of obstructive nephropathy in adults: a comparison between $\mathrm{F}-15$ and a new procedure $\mathrm{F}+10(\mathrm{sp})$ in seated position. Clin Nucl Med 38(6):432-436

Publisher's Note Springer Nature remains neutral with regard to jurisdictional claims in published maps and institutional affiliations. 